Please do not remove this page

RMIT

UNIVERSITY

\title{
Enhancement of human body detection and tracking algorithm based on Viola and Jones framework
}

Krerngkamjornkit, Rapee; Simic, Milan

https://researchrepository.rmit.edu.au/esploro/outputs/9921859651401341/filesAndLinks?institution=61RMIT_INST\&index=null

Krerngkamjornkit, R., \& Simic, M. (2013). Enhancement of human body detection and tracking algorithm based on Viola and Jones framework. Proceedings of 201311 th International Conference on Telecommunications in Modern Satellite, Cable and Broadcasting Services, Serbia (TELSIKS), 115-118. https://doi.org/10.1109/TELSKS.2013.6704903

Document Version: Accepted Manuscript

Published Version: https://doi.org/10.1109/TELSKS.2013.6704903

Repository homepage: https://researchrepository.rmit.edu.au

(C) 2013 IEEE

Downloaded On 2023/04/26 23:18:51 +1000 
Thank you for downloading this document from the RMIT Research Repository.

The RMIT Research Repository is an open access database showcasing the research outputs of RMIT University researchers.

RMIT Research Repository: http://researchbank.rmit.edu.au/

\section{Citation:}

Krerngkamjornkit, R and Simic, M 2013, 'Enhancement of human body detection and tracking algorithm based on Viola and Jones framework', in B. D. Milovanovic (ed.) Proceedings of 2013 11th International Conference on Telecommunications in Modern Satellite, Cable and Broadcasting Services, Serbia (TELSIKS), Serbia, 16 - 19 October 2013, pp. 115-118.

See this record in the RMIT Research Repository at:

http://researchbank.rmit.edu.au/view/rmit:23722

Version: Accepted Manuscript

Copyright Statement: (C) 2013 IEEE

Link to Published Version:

http://dx.doi.org/10.1109/TELSKS.2013.6704903 


\title{
Enhancement of Human Body Detection and Tracking Algorithm based on Viola and Jones Framework
}

\author{
Rapee Krerngkamjornkit ${ }^{1}$, Milan Simic ${ }^{2}$
}

\begin{abstract}
Computer vision application for the human body detection is a fundamental research problem in the areas such as surveillance, search and rescue missions, autonomous driving and other. Building an effective human body detector algorithm is a complex task due to articulation, clothing and illumination conditions. This paper evaluates human body detection and tracking methodology based on the Viola and Jones framework. Presented enhancement aims to address some of the drawbacks from their work which are, sensitivity to noise, poor performances with the complex backgrounds and limits on the scale and rotation.
\end{abstract}

Keywords - Human Body Detection, Pattern Recognition, Vision System.

\section{INTRODUCTION}

Human body detection is the next step after the development of successful face detection algorithms. Humans have been proven to be difficult objects to detect because of the wide variety in appearances, due to articulation, clothing and illumination conditions that are common to outdoor scenes.

A lot of work has been done in recent years on human detection. Previous methods differ in three perspectives:

1. first, use of different features such as edge, haar features and gradient orientation features;

2. second, use of different classifiers such as Nearest Neighbour, Neural Network, Support Vector Machine (SVM) and Adaboost;

3. third, treating the image region as a whole, or detect each part first, and then combine them by these parts' geometrically configurations. [1].

In most approaches, human body detection goes through two stages: segmentation of moving target and classification of human body. In segmentation stage, optical flow method and difference method are often used. For classification, popular methods include template match, feature characterization, cluster analysis, and machine learning approaches using various techniques such as support vector machine, Adaboost, neural network [2].

\footnotetext{
${ }^{1}$ Rapee Krerngkamjornkit is with the School of Aerospace, Mechanical and Manufacturing Engineering, RMIT University, Australia, E-mail: r.krerngkamjornkit@student.rmit.edu.au

${ }^{2}$ Milan Simic is with the School of Aerospace, Mechanical and Manufacturing Engineering, RMIT University, Australia, E-mail: milan.simic@rmit.edu.au
}

The detection of moving human bodies from live videos, especially from videos taken by a moving camera, is not a trivial task. There appear to be two leading approaches. One method uses a single detection window analysis, while the other uses a parts-based approach. Within each method, different authors offer various features and different classifiers to handle the problem [3].

\section{RELATED WORK}

The single-detection-window approach, the work of Papgeorgiou and Poggio [3] uses Haar-based representation, combined with a polynomial SVM. The work of Gavrila and Philomin [4] compare edge images to an exemplar dataset using the chamfer distance. Dalal and Triggs [5] used the single window approach with a dense HoG representation that was successfully applied for object representation [6]. Viola et al. [7] extended their Haar-like wavelets to handle space-time information for moving-human detection.

Others have taken a parts-based approach that aims at dealing with the great variability in appearance, due to body articulation. In this approach, each part is detected separately and a human is detected if some, or all of its parts are presented in a geometrically plausible configuration. Felzenswalb \& Huttenlocher [8] use pictorial structure approach where, an object is described by the connection of its parts, and each part represented by Gaussian derivative filters of different scale and orientation. Ioffe \& Forsyth [9] represent parts as projections of straight cylinders and propose efficient ways to incrementally assemble these segments into a full body assembly. Mikolajczyk et al. [10] represent parts as co-occurrences of local orientation features. The system proceeds by detecting features, then parts and eventually humans are detected based on assemblies of parts.

Viola et al. [7] developed a framework for detecting humans in a surveillance environment. The environment assumes that people to be detected are very small and usually have a clear background (road, wall, etc.). In this scenario the detection performance greatly relies on the available motion information.

We choose to work on the enhancement of Viola and Jones Framework. For our application, we need to concentrate on real time, therefore the frame to frame processing time is crucial. The speed of the approach taken by Viola and Jones is its strong point. Our enhancement aims to address some of the drawbacks from their work which are, sensitive to noise, poor performances with the complex backgrounds and limit on scale and rotation. The following sections provide explanation and experimental results of our work. 


\section{Human Body Detection Method}

\section{A. Algorithm Overview}

The success of the Viola-Jones detector [11] illustrated the feasibility of real-time face detection. Their key to success is known for simple and fast-to-compute set of features. They use a machine meta learning algorithm that could perform the computationally complex task of learning offline, as shown in Fig.1. The learning algorithm is called Adaboost [12]. The sacrifice at the feature level, applied here, can make the detector more sensitive to noise [13].

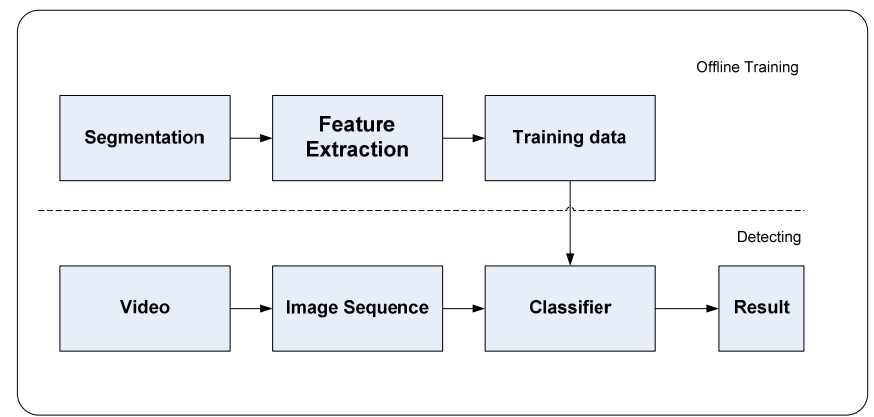

Fig. 1. Human Body Detection Method

\section{B. Viola and Jones Framework}

Viola and Jones use features that are composed of sums of the images (I) within boxes $B(I)$. Sums are weighted by 1 or 1 , and then added together. This yields to the form:

$$
\sum_{k} \delta_{k} B_{k}(I)
$$

Where $\delta i \in\{1,-1\}$ and

$$
B_{k}(I)=\sum_{i=u 1(k)}^{u 2(k)} \sum_{j=11(k)}^{v 2(k)} I_{i j}
$$

Such features are extremely fast to evaluate with a device called an integral image, labelled as $\hat{\boldsymbol{I}}$. The integral image is formed from the images $I$. as shown in Eq. (3)

$$
\hat{\boldsymbol{I}}_{i j}=\sum_{u=1}^{i} \sum_{v=1}^{j} \boldsymbol{I}_{u v}
$$

Where $u$ and $v$ refer to pixel values of an integral image.

This means that any sum within a box can be evaluated with four queries to the integral image. It is easy to check that

$$
\sum_{i=u 1}^{u 2} \sum_{j=v 1}^{v 2} I_{i j}=\hat{I}_{u 2 v 2}-\hat{I}_{u 1 v 2}-\hat{I}_{u 2 v 1}+\hat{I}_{u 1 v 1}
$$

This means that any of the features can be evaluated by a set of integral image queries [9].
In computer vision, segmentation is the process of partitioning a digital image into multiple segments, or sets of pixels. The goal is to simplify, i.e. change the representation of an image into regions as a focus for further investigation. Image segmentation is typically used to locate objects and boundaries such as lines and curves.

The result of image segmentation is a set of segments that collectively cover the entire image, or a set of contours extracted from the image. The set of pixels, in a region, are similar with respect to some characteristic, or computed property, such as colour, intensity, or texture. Adjacent regions are different with respect to the same characteristics. The task of the segmentation is to split the image into several regions based on colour, motion or texture information [14].

\section{Training}

One strategy to get a better classifier is to combine multiple classifiers. A natural approach is to train a classifier on a dataset; then train a new classifier, weight each example to train the new classifier to get examples right. If the previous classifier was wrong; repeat this number of times. The final result is a weighted combination of the outputs of all these classifiers. This general process is called boosting.

Boosting provides quite successful classifiers. Process can continue after the training error rate falls to zero. The number of boosting rounds is usually chosen with a validation set (one continues to boost until the error on the validation set rises).

\section{E. Classification}

Classification is determining whether, or not, the image data contains some specific object, feature, or activity. This task can be solved robustly and without effort by a human, but it is not satisfactorily. It is a difficult computer vision task. Methods used for dealing with this problem can solve specific objects recognition, such as simple geometric objects, human faces, printed, hand-written characters, and vehicles in particular situations. They are typically described in terms of well-defined illumination, background and pose of the object relative to the camera.

Classification is basically a pattern recognition problem of assigning an object to a class, or a set of classes. Thus the output of the recognition system can be an integer label. The task of the classifier is to partition the feature space into classlabelled decision regions [14].

\section{EXPERIMENT}

In our experiment, we have created a simple upper body tracking system that automatically detects and tracks a single upper body. Object detection and tracking are important in many computer vision applications including activity recognition, automotive safety, surveillance and autonomous driving. Our, upper body tracking system, divided the complex task into three separate problems, as shown in Fig. 2: Detect, Identify and Track upper body. 


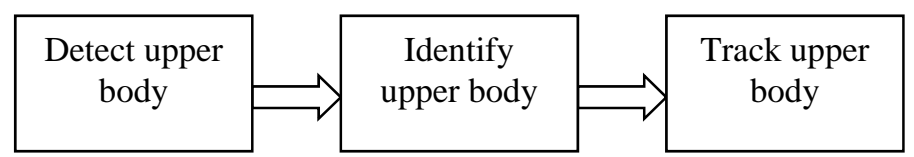

Fig. 2. Upper body detection and tracking

\section{A. Detect a face to track}

Before begin tracking a body, we need to detect it. We used the cascade object detector to identify the location of an upper body in a video frame. This detector uses the Viola-Jones detection algorithm and a trained classification model for detection. We try to track an upper body across successive video frames. Head movements could cause a loss of tracking. This limitation is due to the type of trained classification model used for detection. To avoid this issue, and because the performing face detection, for every video frame, is computationally intensive, we use a simple feature for tracking.

\section{B. Identify facial features to track}

Once the upper body is located in the video, we need to identify a feature that will help us to track it. For example, we can use the shape, texture, or colour. The feature needs to be chosen that is unique to the object and remains invariant even when the object moves. In this example, we use skin tone as the feature to track. The skin tone provides a good deal of contrast between the upper body and the background and does not change as the face rotates or moves. It depends on the environmental light conditions.

\section{Track the upper body}

With the skin tone selected as the feature to track, we can use a Histogram Based Tracker for tracking, providing the capability to track an object using a histogram of pixel values. In this experiment, the Hue channel pixels are extracted from the region of detected upper body. These pixels are used to initialize the histogram for the tracker. It tracks the object over successive video frames using this histogram. The algorithm for upper body detection is shown in the Table 1. It is noted that the method sometimes produces false positive. In Fig. 3a. and 3b., two upper bodies were found. One is from the right hand size on the photo and the second one is the correct upper body.

Many factors, such as file size, may have an impact on the result. The algorithm needs to be configured according to the design requirements, such as, what should be detected, video or image size, image type, or light. For example, when we have changed video file for smaller size of human, we noticed poor tracking results. Hue channel data has to be adjusted for enough contrast between the upper body region and the background.

Adjusting the size of detector has improved results. The size of detector was too small for the original file size of 1019 x 566 pixels, so that algorithm produced a false detection. If we increase the size of the detector, the false detection decreases.

TABLE I

UPPER BODY DETECTION ALGORITHM

\section{Detect upper body}

- $\quad$ Create a cascade detector object.

- Read a video frame and run the detector.

- Draw the returned bounding box around the detected upper body.

\section{Identify upper body features to track}

- Get the upper body information by extracting the Hue from the video frame.

- $\quad$ Converted to the Hue, Saturation and Value (HSV) colour space.

- Display the Hue Channel data and draw the bounding box around the upper body.

- $\quad$ Detect the upper body within the region.

3. Track upper body

- Create a tracker object.

- Initialize the tracker histogram using the Hue channel pixels.

- $\quad$ Create a video player object for displaying video frames.

- $\quad$ Track the upper body over successive video frames until the video is finished.

- $\quad$ Extract the next video frame.

- Track using the Hue channel data.

- Insert a bounding box around the object being tracked.

- Display the annotated video frame using the video player object.

The behaviour of these properties is affected by the scale factor. We managed to increase the size of search area and the scale factor. The scale factor determines the quantization of the search window sizes. The size of search area can be varied, and is used to speed up the computational time.

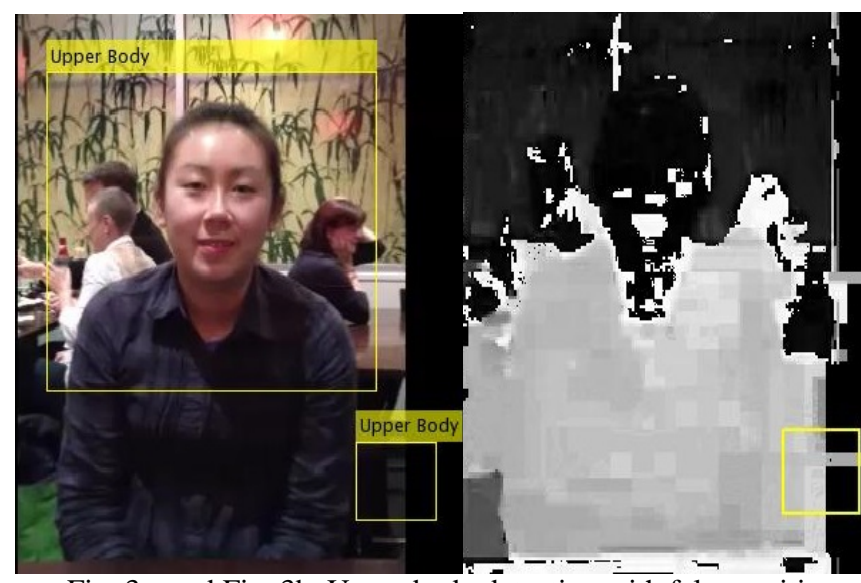

Fig. 3a. and Fig. 3b. Upper body detection with false positive

In Fig. 4a. and 4b., we have shown better result obtained. Table 2 shown results of Viola and Jones compare to our method. The main reason for our method to work better than 
Viola and Jones is because we choose simple feature set and lower complexity (lower number of features used) for the upper body detection.

\section{TABLE II}

COMPARISON OF UPPER BODY DETECTION ALGORITHMS

\begin{tabular}{|l|l|l|l|}
\hline Method & $\begin{array}{l}\text { Detection } \\
(\%)\end{array}$ & $\begin{array}{l}\text { False } \\
\text { positive (\%) }\end{array}$ & $\begin{array}{l}\text { Processing } \\
\text { time (ms) }\end{array}$ \\
\hline $\begin{array}{l}\text { Viola \& } \\
\text { Jones }\end{array}$ & 71.2 & 0.012 & 30 \\
\hline Our method & 84.6 & 0.007 & 28 \\
\hline
\end{tabular}

However the above algorithm was tested for only single object (person). It will be slightly challenging to run the detector on multiple objects from video file and observe the result. That is another research objective. We will use similar approach to test and optimise algorithms.

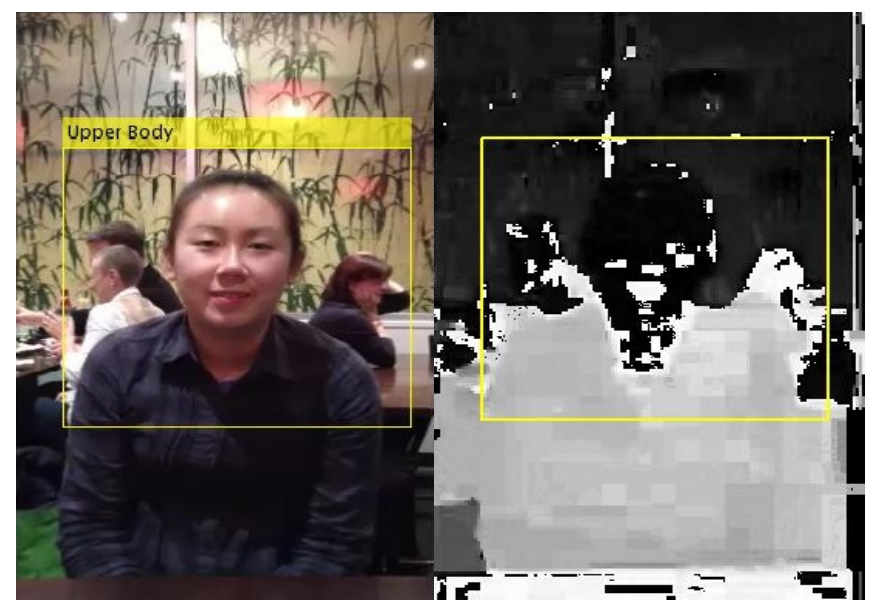

Fig. 4a. and Fig. 4b. Upper body detection improved

\section{CONCLUSIONS}

We are conducting research in vision and image recognition for the applications like advanced manufacturing, surveillance, search and rescue missions, autonomous driving and other. Some first, initial results are presented here.

There is always space for the improvements. Future work will be to adjust parameters and modify functions in order to have more accurate upper body detector. This includes:

1. Detection: Associating detections over time, such as size, shape, and colour.

2. Tracking: Varying parameters for tracking stage, assignment, deletion of track, add model to the original algorithm for "constant acceleration" or add "Kalman" filter for every object, or other type of filter such as "Particle" filter.

\section{REFERENCES}

Z. Qiang, M. C. Yeh, C. Kwang-Ting, and S. Avidan, "Fast Human Detection Using a Cascade of Histograms of Oriented Gradients," in Computer Vision and Pattern Recognition, 2006 IEEE Computer Society Conference on, 2006, pp. 14911498.
[2] X. Fen and G. Ming, "Human detection and tracking based on HOG and particle filter," in Image and Signal Processing (CISP), 2010 3rd International Congress on, 2010, pp. 1503-1507.

C. Papageorgiou and T. Poggio, "A Trainable System for Object Detection," International Journal of Computer Vision, vol. 38, pp. 15-33, 2000.

D. M. Gavrila and V. Philomin, "Real-Time Object Detection for "Smart" Vehicles," in IEEE Conference on Computer Vision and Pattern Regconition, 1997, pp. 193-199.

N. Dalal and B. Triggs, "Histograms of oriented gradients for human detection," in Conference on Computer Vision and Pattern Recognition, 2005, pp. 886-893.

D. Lowe and G. Lowe, "Distinctive Image Features from Scale-Invariant Keypoints," International Journal of Computer Vision, vol. 60, pp. 91-110, 2004.

[7] V. P., J. M., and S. D., "Detecting pedestrains using patterons of motion and apperance," in International Conference on Computer Vision, 2003.

[8] P. Felzenszwalb, F. Huttenlocher, D. Huttenlocher, and P. Huttenlocher, "Pictorial Structures for Object Recognition," International Journal of Computer Vision, vol. 61, pp. 55-79, 2005.

[9] S. Ioffe and D. A. Forsyth, "Probabilistic Methods for Finding People," International Journal of Computer Vision, vol. 43, pp. 45-68, 2001.

[10] K. Mikolajczyk, C. Schmid, and A. Zisserman, "Human Detection Based on a Probabilistic Assembly of Robust Part Detectors," in Computer Vision - ECCV 2004. vol. 3021, T. Pajdla and J. Matas, Eds., ed: Springer Berlin Heidelberg, 2004, pp. 69-82.

P. Viola and M. Jones, "Rapid object detection using a boosted cascade of simple features," in Computer Vision and Pattern Recognition, 2001. CVPR 2001. Proceedings of the 2001 IEEE Computer Society Conference on, 2001, pp. I-511-I-518 vol.1.

[12] Y. Freund and R. E. Schapire, "A Decision-Theoretic Generalization of On-Line Learning and an Application to Boosting*," Journal of computer and system sciences, vol. 55, pp. 119-139, 1997.

[13] H. Sugano, R. Miyamoto, and Y. Nakamura, "Optimized parallel implementation of pedestrian tracking using HOG features on GPU," in Ph.D Research in Microelectronics and Electronics (PRIME), 2010 Conference on, 2010, pp. 1-4.

[14] R. Krerngkamjornkit and M. Simic, "Human body detection in search and rescue operation conducted by unmanned aerial vehicles," in 3rd International Conference on Advances in Materials and Manufacturing Processes, Beihai, China, 2012, pp. 1077-1085. 